\title{
Pneumoencéfalo difuso após fratura de maxila: relato de caso
}

\author{
Cristóvão Marcondes de Castro RODRIGUES'; Mirlany Mendes \\ Maciel OLIVEIRA'; Daniela Meneses SANTOS ${ }^{1}$; Cláudia Jordão \\ SILVA2; Lair Mambrini FURTADO2; Jonas Dantas BATISTA2
}

1 - Residente em Cirurgia e Traumatologia Bucomaxilofacial - Hospital de Clínicas da Universidade Federal de Uberlândia (HC-UFU), Uberlândia, Minas Gerais, Brasil; 2 - Preceptor da Residência em Cirurgia e Traumatologia Bucomaxilofacial - Hospital de Clínicas da Universidade Federal de Uberlândia (HC-UFU), Uberlândia, Minas Gerais, Brasil.

\section{Resumo}

Objetivo: O presente trabalho teve por objetivo apresentar caso incomum de pneumoencéfalo difuso em massa encefálica decorrente de trauma simples de maxila Lefort I bilateral. Material e método: Paciente T.S, gênero masculino, 35 anos, vítima de acidente motociclístico apresentando ao exame clínico extraoral abrasões em região frontal e geniana esquerda e ferida corto contusa em região frontal apresentava alteração oclusal, mobilidade crânio-caudal e latero-lateral de maxila. Ao exame de tomografia computadorizada confirmou-se a presença de fratura de maxila Le Fort I e fratura de parede anterior do seio frontal e observou-se também a presença de pneumoencéfalo difuso em região da massa encefálica e grande quantidade de enfisema nos espaços faciais. Resultados: Sob orientação da equipe de neurocirurgia foi iniciado antibioticoterapia com uso de Ceftriaxona $1000 \mathrm{mg}$ duas vezes ao dia por sete dias e realizado tomografia de crânio controle foi onde se observou regressão completa do pneumoencéfalo. Após instalação de barra de Erich realizou-se a redução e fixação da fratura Lefort I bilateral sob anestesia geral sem intercorrências. Conclusão: Exames de imagem, como a tomografia computadorizada, são indispensáveis para uma adequada avaliação e diagnóstico das condições intracraniana. Por mais que o pneumoencéfalo possua, na maioria dos casos, resolução espontânea um acompanhamento rigoroso é fundamental para a melhor condução dos casos.

PALAVRAS-CHAVE: Maxila; Traumatismos Faciais; Pneumocefalia.

AUTOR PARA CORRESPONDÊNCIA

Mirlany Mendes Maciel Oliveira

Departamento de Cirurgia e Traumatologia Bucomaxilofacial e Implantodontia

Endereço: Av. Pará, 1748, Umuarama, Uberlândia - MG, 38405-320 Bloco 4T, Uberlândia Minas Gerais - MG

Telefone: (34) 997906251 / E-mail: mirlany.mendes@gmail.com 


\section{Introdução}

O trauma crânio encefálico (TCE) é uma agressão ao cérebro, causada por uma força física externa, que acarreta lesão anatômica e/ou comprometimento funcional do couro cabeludo, crânio, meninges ou encéfalo ${ }^{1}$. O pneumoencéfalo é uma possível complicação decorrente do TCE, sendo a principal causa de morbimortalidade relacionada ao trauma, no Brasil ${ }^{1,2}$.

O pneumoencéfalo é uma coleção de ar na cavidade intracraniana, geralmente circunscrito e restrito em uma determinada localização intracraniana, sendo a maioria dos casos benigno, assintomático e de resolução espontânea, entretanto quando volumoso, causa o efeito de massa com sinais e sintomas de hipertensão intracraniana ${ }^{2,3}$. Um quadro de pneumoencéfalo difuso é muito raro, geralmente quando ocorrido sua localização mais comum é espaço subaracnóideo, tendo resolutividade mais rápida sem necessidade de abordagem cirúrgica ${ }^{2,4}$. Logo, existe relação íntima entre traumatismo crânio encefálico, pneumoencéfalo e os traumatismos faciais considerando a proximidade anatômicas das estruturas associadas a esses fenômenos ${ }^{4,5}$.

Os principais sintomas que podem ocorrer em consequência da hipertensão intracraniana são: cefaléia frontal, que piora quando o paciente assume posição sentada ou ortostática, convulsão, diminuição do nível de consciência, náusea, vômito, tontura e hemiparesia ${ }^{6}$. A duração e a intensidade dos sintomas estão relacionadas com a quantidade de ar intracraniano ${ }^{6}$. O pneumoencéfalo e os sinais e sintomas do TCE tende a regredir espontaneamente ente $2-3$ dias $^{6,7}$.

Radiograficamente, o pneumoencéfalo pode ser detectado por radiografias de crânio desde que, tenha um volume de ar superior a $2 \mathrm{ml}^{8,9}$. Sendo a tomografia de crânio o exame de imagem padrão ouro'. 
Esse artigo objetivou, relatar um caso de um paciente vítima com TCE leve, apresentando uma simples fratura do complexo maxilo facial Lefort I e que exibiu a rara condição de um quadro de pneumoencéfalo difuso.

\section{Relato de caso}

Paciente T.S, gênero masculino, 35 anos, vítima de acidente motociclístico foi encaminhado ao Hospital de Clínicas da Universidade Federal de Uberlândia (HC - UFU) com escoriações em face. Ao exame clínico extraoral notou-se abrasões em região frontal e geniana esquerda e ferida corto contusa em região frontal. Ao exame clínico intraoral, paciente apresentava alteração oclusal, mobilidade crânio-caudal e latero-lateral de maxila e laceração em gengiva inserida na região anterior da maxila. A suspeita clínica era de fratura de maxila Le Fort I. As lacerações extras e intraorais foram suturadas e foram solicitados exames de imagem de tomografia computadorizada helicoidal da face. Ao exame tomográfico, confirmou-se presença de fratura Lefort I, fratura de parede anterior do seio frontal e observou-se também a presença de pneumoencéfalo difuso em região da massa encefálica nos cortes axiais (Figuras 1A e 1B) e nos cortes sagitais (Figuras 1C e 1D) e grande quantidade de enfisema nos espaços faciais, visto que a fratura de maxila se encontrava com pouco deslocamento. Logo a equipe da neurocirurgia foi contactada para parecer clínico.

No parecer da equipe da neurocirurgia, o exame de imagem não apresentava sinais de desvio de linha média, ausência de efeito expansivo de massa, paciente encontrava-se Glasgow 15, as pupilas estavam isocóricas e fotorreagentes, níveis de força muscular preservados, ausência de náusea ou vômitos. Foi sugerido aguardar regressão do pneumoencéfalo para abordagem cirúrgica, repetir tomografia controle de 48 horas, avaliar a possibilidade de fístula liquórica ativa e manter paciente com Ceftriaxona $1000 \mathrm{mg}$ duas vezes ao dia por sete dias. A tomografia de crânio controle foi realizada, onde se observou regressão 
completa do pneumoencéfalo nos cortes axiais (Figuras 2A e 2B) e nos cortes sagitais (Figuras 2C e 2D). $\mathrm{Na}$ reavaliação clínica não foi observado presença de fístula liquórica visto que paciente foi submetido à pesquisa do sinal halo e ao estímulo da pressão intracraniana (inclinação do corpo e realização da manobra de Valsalva), o que permitiu a abordagem cirúrgica pela equipe da bucomaxilofacial.

Após instalação de barra de Erich e redução e fixação da fratura Lefort I bilateral sob anestesia geral sem intercorrências, paciente permaneceu internado por mais quatro dias para manutenção da dose de Ceftriaxona sugerida pela equipe da neurocirurgia.

Na alta hospitalar, na prescrição domiciliar o paciente foi orientado quanto ao uso dos analgésicos e à higiene oral devido ao acesso cirúrgico para tratamento da fratura em face. Paciente seguiu em acompanhamento ambulatorial por quarenta e cinco dias devido à fratura de face, removida a barra de Erich e alta ambulatorial.

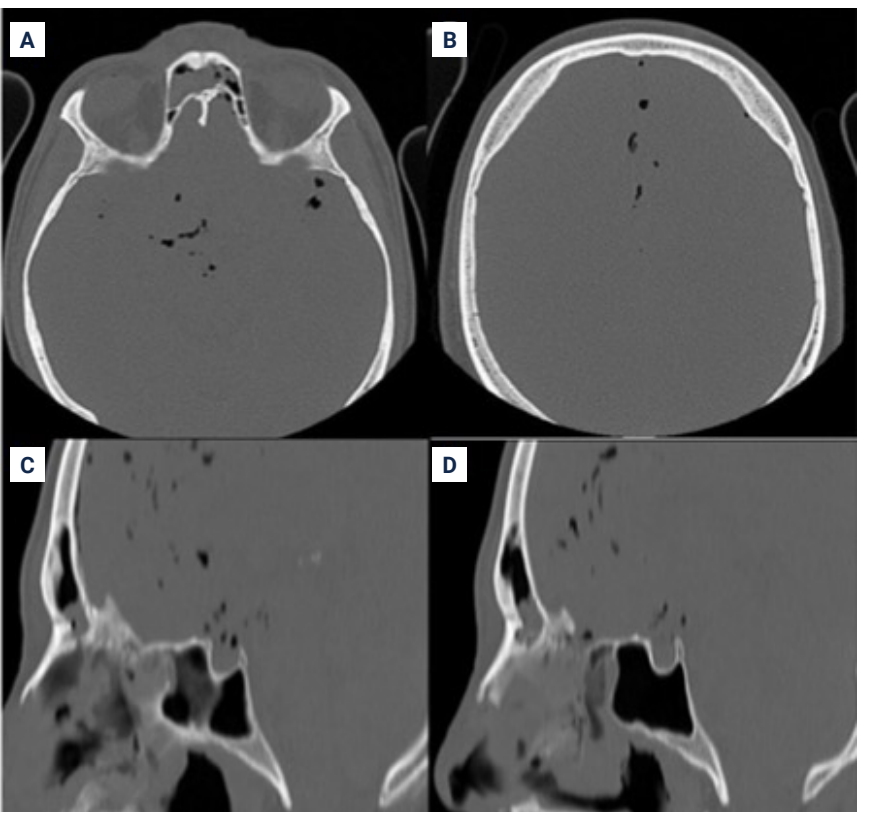

FIGURA 1 - Cortes axiais de tomografia computadorizada apresentando evidências de pneumoencéfalo difuso (A e B). Cortes sagitais de tomografia computadorizada apresentando evidências de pneumoencéfalo difuso (C e D)

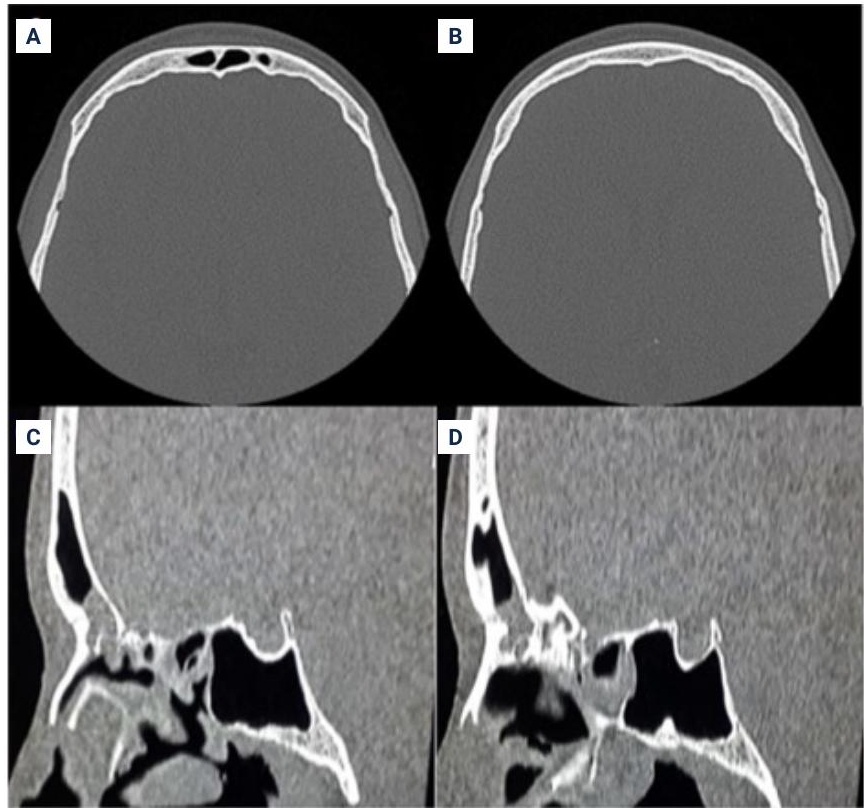

FIGURA 2 - Cortes axiais de tomografia computadorizada apresentando evidências de regressão completa do quadro de pneumoencéfalo difuso (A e B). Cortes sagitais de tomografia computadorizada apresentando evidências de regressão completa do quadro de pneumoencéfalo difuso (C e D) 


\section{Discussão}

As causas de pneumoencéfalo são diversas. As mais comuns são devido a TCE e traumatismo facial ${ }^{10,11}$. A intensidade e duração dos sintomas dependem da distribuição intracraniana de ar e estão relacionadas ao volume de ar. Quando pequeno é assintomático ou apresenta sinais e sintomas inespecíficos, sendo às vezes diagnosticado após a realização de exames de imagens de rotina $^{12-14}$. Como no caso descrito, paciente não apresentou alteração na escala de consciência durante o período de internação, assim como nenhum dos sintomas comumente causados por um característico pneumoencéfalo hipertensivo como náusea, cefaléia, alteração de consciência e alterações pupilares.

A tomografia computadorizada é o exame de imagem padrão ouro para diagnóstico de fraturas de terço médio facial e utilizado para diagnosticar alterações de fundo neurológico ${ }^{13}$. A tomografia computadorizada requer apenas $0,55 \mathrm{~mL}$ de ar para que o pneumoencéfalo seja identificado, já as radiografias de crânio requerem no mínimo $2 \mathrm{~mL}$ de $\mathrm{ar}^{8}$. Como no caso exposto, por ser tratar de uma fratura do terço médio de face simples com pouco deslocamento indicativo de impacto de baixa energia e sem alterações neurológicas presentes, comumente seria proposto incidência radiográfica de Waters o que pode impossibilitar o achado imaginológico de alterações cranioencefálicas, como o penumoencéfalo difuso. A ressonância magnética também pode ser um exame de imagem indicado, contudo a sua sensibilidade é menor que a TC no diagnóstico do pneumoencéfalo ${ }^{15}$.

Em relação ao diagnóstico diferencial no que se diz respeito aos achados imaginológicos do pneumoencéfalo é importante saber que o ar tem um coeficiente de Hounsfield de -1000 , sendo que a gordura intracraniana, tem uma densidade muito maior (-90 $\mathrm{UH})$ podendo parecer hipodensa na TC levando ao profissional confundir com pneumoencéfalo ${ }^{16}$.

O trauma é o fator etiológico predominante do pneumoencéfalo, correspondendo $67-74 \%$ dos $\operatorname{casos}^{13}$. Nos traumas faciais, devido 
as altas energias dissipadas, características desses acidentes, é comum associação de pneumoencéfalo e fístula liquórica, que nada mais é que uma lesão na membrana da aracnóide, dura-máter, osso e mucosa, que resulta em fluxo extracraniano de líquor. As fístulas são classificadas de forma didática em fístulas de origem nasal e de origem otológica ${ }^{17}$.

Em 75\% dos casos de fístula liquórica (rinoliquorreia, otoliquorreia), apresenta resolução espontânea dentro de uma semana do início dos sintomas, e os mesmos podem regredir espontaneamente ou serem aliviados pelo repouso no leito, posição supina ou Trendelemburg, hidratação e analgésicos ${ }^{18}$. No presente caso, não foi constatado fístula liquórica, realizando teste de Valsalva e beta -2-tranferrina, paciente não apresentou sinais de otorragia e rinoliquorréia, durante período de internação.

O tratamento dos quadros de pneumoencéfalo inclui desde a conduta conservadora para casos de pneumoencéfalo pequeno, assintomático e sem efeito de massa, até procedimentos cirúrgicos para remoção do ar intracraniano quando apresenta efeito de massa, laceração da dura-máter, correção do defeito congênito na base do crânio e profilaxia contra meningite pós-traumática ${ }^{15}$. O tratamento depende da sua etiologia, quadro neurológico, da extensão, volume e progressão da coleção de ar. Na maioria dos casos o volume de ar intracraniano é pequeno, assintomático e apresenta resolução espontânea ${ }^{16,19}$. O tratamento conservador inclui repouso absoluto no leito em decúbito dorsal, evitar manobras que aumente a pressão intracraniana, oxigenioterapia, analgesia e antibioticoterapia sistêmica com ciclosporina de terceira geração como ceftriaxone ou cefotaxima, na suspeita e/ou profilaxia da meningite, sendo necessário controle clínico rigoroso e tomografia computadorizada de controle ${ }^{20-22}$. Assim, como corroborado na literatura, no caso retratado o paciente foi submetido a repouso absoluto, medicado com cefetriaxone $1000 \mathrm{mg} 2 \mathrm{x}$ ao dia por 7 dias, como prevenção de quadro de meningite e submetido a oxigenioterapia com cabeceira do leito sempre elevada. 


\section{Conclusões}

O caso em questão nos faz inferir sobre os protocolos dos exames de imagens em casos de pacientes politraumatizados, uma vez que incidências radiográficas convencionais, como incidência de Waters, podem levar ao subdiagnóstico de alterações encefálicas, sendo que os exames de imagem como a tomografia computadorizada são indispensáveis para uma adequada avaliação e diagnóstico das condições intracranianas. Mesmo que o fenômeno de pneumoencéfalo seja recorrente nesses casos e em sua maioria de resolução espontânea, o acompanhamento rigoroso é mandatório para melhor condução dos casos, mesmo nos episódios que traumas faciais estejam mais evidentes do que nas estruturas cranianas. Portanto, as vítimas de acidentes crânio faciais, devem ser submetidos a exames clínicos minuciosos, considerando que na grande maioria das vezes esses acidentes têm como fatores etiológicos trauma de alta energia, que podem levar a comprometimento das estruturas craniofaciais.

\section{Referências}

1 - Melo JRT, Silva RA, Moreira ED. Características dos pacientes com trauma Cranioencefalico na Cidade de Salvador, Bahia, Brasil. Arq Neuropsiquiatr. 2004; 62(3-A): 711-5.

2 - David CA. Traumatismo Cerebral. In: Jones HR (ed). Neurologia de Netter. Porto Alegre: Artmed; 2006. p. 672-85.

3 - Leong KM, Vijayananthan A, Sia SF, Waran V. Pneumocephalus: an uncommon finding in trauma. Med J Malaysa. 2008; 63(3): 256-258.

4 - Aguilar-Shea AL, Mañas-Gallardo N, Romero-Pisonero E. Posttraumaticpneumocephalus. Int J Emerg Med. 2009; 2(2): 129-130.

5 - Lee JS, Park YS, Kwon JT, Suk JS. Spontaneous pneumocephalus associated with pneumosinusdilatans. J Korean Neurosurg Soc. 2010; 47(5): 395-398.

6 - Dabdoub CB, Salas G, Silveira EN, Dabdoub CF. Review of the management of pneumocephalus. Surg Neurol Int. 2015; 6(2): 155-159.

7 - Pop PM, Thompson JR, Zinke DE, Hasso AN, Hinshaw DB. Tension pneumocephalus. J Comput Assist Tomogr. 1982; 6(5): 894-901.

8 - Dabdoub CB, Salas G, Silveira EM, Dabdoub CF. Review of the management of pneumocephalus. Surg Neurol Int. 2015; 6:155. 
9- Karavelioglu E, Eser O, Haktanir A. Pneumocephalus and pneumorrhachis after spinal surgery: case report and review of the literature. Neurol Med Chir (Tokyo). 2014; 54(5): 405-407.

10 - Marakham JW. The clinical features of pneumocephalus based upon a survey of 284 cases with report of 11 additional cases. Acta Neurochir (Wien). 1967; 16(1): 1-78.

11 - Steudel WI, Hacker H. Acute intracranial pneumocephalus: prognosis and management: a retrospective analysis of 101 cases. Neurosurg Rev. 1989; 12(1): 125-136.

12 - Shehu BB, Ismail NJ, Hassan I. Management of pneumoencephalus in a resource limited environment: Review from sub-Saharan Africa. BrainInj. 2007; 21(7): 1217-1223.

13 - Pillai P, Sharma R, MacKenzie L, Reilly EF, Beery PR, Papadimos TJ, Stawicki SPA. Traumatic tension pneumocephalus: two cases and comprehensive review of literature. Int J Crit Illn Inj Sci. 2017; 7(1): 58-64.

14 - Das JM, Bajaj J. Pneumocephalus. Stat Pearls Publishing. 2019.

15 - Chandran TH, Prepageran N, Philip R, Gopala K, Zubaidi AL, Jalaludin MA. Delayed spontaneous traumatic pneumocephalus. Med J Malasya. 2007; 62(5): 411-412.

16 - Kapoor T, Shetty P. Pneumocephalus. J Emerg Med. 2008; 35(4): 453-454

17 - Zweig JL, Carrau RL, Celin SE, Schaitkin BM, Pollice PA, Snyderman $\mathrm{CH}$, Kassam A, Hegazy H. Endoscopic repair of cerebrospinal fluid leaks to the sinonasal tract: predictors of success. Otolaryngol Head NeckSurg. 2000; 123(3): 195-201.

18 - Dabdoub CB, Salas G, Silveira EN, Dabdoub CF. Review of the management of pneumocephalus. Surg Neurol Int. 2015; 6: 155-159.

19 - González-Bonet LG, Goig-Revert FA, Rodríguez-Mena R, BarciaMariño C. Neumoencefalo a tensión tras herida en cuero cabelludo en portadora de válvula de derivación ventrículo-peritoneal: caso clínico y revisión de la literatura. Neurocirugía. 2009; 20(2): 152-158.

20 - Pillai P, Sharma R, MacKenzie L, Reilly EF, Beery II PR, Papadimos TJ, et al. Traumatic tension pneumocephalus: two cases and comprehensive review of literature. OPUS 12 Scientist. 2010; 4(1): 6-11.

21 - Paiva WS, Andrade AF, Figueiredo EG, Amorim RL, Prudente M, Teixeira MJ. Effects of hyperbaric oxigenation therapy on symptomatic pneumocephalus. Ther Clin Risk Manag. 2014; 10(6): 769-773.

22 - Pereira CU, Dezena RA, Meguins LC, Teixeira MPS. Pneumoencéfalo. Revisão da Literatura. J Bras Neurocirurg. 2015; 26(1): 47-56. 


\title{
Pneumoencefalo diffusion after maxila fracture: case report
}

\begin{abstract}
Objective: The present study aimed to present an unusual case of diffused pneumocephalus in encephalic mass resulting from simple bilateral Lefort I maxillary trauma. Case report: TS patient, male, 35 years old, motorcycle accident victim presenting at the extraoral clinical examination abrasions in the frontal and left genic region and short blunt wound in the frontal region presented occlusal alteration, cranio-caudal and lateral-lateral mobility of the maxilla , computed tomography examination confirmed the presence of Le Fort I maxilla fracture and anterior wall fracture of the frontal sinus, and the presence of diffused pneumocephalus the region of the encephalic mass and a large amount of emphysema in the facial spaces was also observed. Results: Under the guidance of the neurosurgery team, antibiotic therapy was started using Ceftriaxone $1000 \mathrm{mg}$ twice daily for seven days and a control cranial tomography was performed and a complete regression of pneumoencephalus was observed. After Erich bar installation, bilateral Lefort I fracture reduction and fixation were performed under general anesthesia without complications. Conclusion: Imaging examinations such as computed tomography are indispensable for an adequate evaluation and diagnosis of intracranial conditions. Although pneumoencephalus had in most cases spontaneous resolution, strict follow-up is essential for better case management.
\end{abstract}

KEYWORDS: Maxilla, Facial Injuries, Pneumocephaly.

\section{Como citar este artigo}

Rodrigues CMC, Oliveira MMM, Santos DM, Silva CJ, Furtado LM, Batista JD. Pneumoencéfalo difuso após fratura de maxila: relato de caso. Rev Odontol Bras Central 2021; 30(89): 171-179. DOI: 